\title{
Genome-scale constraint-based modeling of Geobacter metallireducens
}

\author{
Jun Sun*1, Bahareh Sayyar ${ }^{2}$, Jessica E Butler ${ }^{3}$, Priti Pharkya1, Tom R Fahland ${ }^{1}$, \\ Iman Famili ${ }^{1}$, Christophe H Schilling ${ }^{1}$, Derek R Lovley ${ }^{3}$ and \\ Radhakrishnan Mahadevan*2
}

\author{
Address: ${ }^{1}$ Genomatica Inc., 10520 Wateridge Circle, San Diego, CA, USA, ${ }^{2}$ Department of Chemical Engineering and Applied Chemistry, \\ University of Toronto, Toronto, Ontario, Canada and ${ }^{3}$ Department of Microbiology, University of Massachusetts, Amherst, MA, USA \\ Email: Jun Sun* - jsun@genomatica.com; Bahareh Sayyar - bahareh.sayyar@utoronto.ca; Jessica E Butler - jbutler@microbio.umass.edu; \\ Priti Pharkya - ppharkya@genomatica.com; Tom R Fahland - tfahland@genomatica.com; Iman Famili - ifamili@genomatica.com; \\ Christophe H Schilling - cschilling@genomatica.com; Derek R Lovley - dlovley@microbio.umass.edu; \\ Radhakrishnan Mahadevan* - krishna.mahadevan@utoronto.ca \\ * Corresponding authors
}

Published: 28 January 2009

BMC Systems Biology 2009, 3:15 doi:10.1186/1752-0509-3-15

This article is available from: http://www.biomedcentral.com/I752-0509/3//5

(c) 2009 Sun et al; licensee BioMed Central Ltd.

This is an Open Access article distributed under the terms of the Creative Commons Attribution License (http://creativecommons.org/licenses/by/2.0), which permits unrestricted use, distribution, and reproduction in any medium, provided the original work is properly cited.

\begin{abstract}
Background: Geobacter metallireducens was the first organism that can be grown in pure culture to completely oxidize organic compounds with Fe(III) oxide serving as electron acceptor. Geobacter species, including $G$. sulfurreducens and $G$. metallireducens, are used for bioremediation and electricity generation from waste organic matter and renewable biomass. The constraint-based modeling approach enables the development of genomescale in silico models that can predict the behavior of complex biological systems and their responses to the environments. Such a modeling approach was applied to provide physiological and ecological insights on the metabolism of $G$. metallireducens.
\end{abstract}

Results: The genome-scale metabolic model of $G$. metallireducens was constructed to include 747 genes and 697 reactions. Compared to the $G$. sulfurreducens model, the $G$. metallireducens metabolic model contains I I 8 unique reactions that reflect many of $G$. metallireducens' specific metabolic capabilities. Detailed examination of the $G$. metallireducens model suggests that its central metabolism contains several energy-inefficient reactions that are not present in the $G$. sulfurreducens model. Experimental biomass yield of $G$. metallireducens growing on pyruvate was lower than the predicted optimal biomass yield. Microarray data of $G$. metallireducens growing with benzoate and acetate indicated that genes encoding these energy-inefficient reactions were up-regulated by benzoate. These results suggested that the energy-inefficient reactions were likely turned off during $G$. metallireducens growth with acetate for optimal biomass yield, but were up-regulated during growth with complex electron donors such as benzoate for rapid energy generation. Furthermore, several computational modeling approaches were applied to accelerate $G$. metallireducens research. For example, growth of $G$. metallireducens with different electron donors and electron acceptors were studied using the genome-scale metabolic model, which provided a fast and cost-effective way to understand the metabolism of $G$. metallireducens.

Conclusion: We have developed a genome-scale metabolic model for $G$. metallireducens that features both metabolic similarities and differences to the published model for its close relative, $G$. sulfurreducens. Together these metabolic models provide an important resource for improving strategies on bioremediation and bioenergy generation. 


\section{Background}

Geobacter species are environmentally significant because of their capacity for dissimilatory Fe(III) reduction [1]. They can conserve energy for growth by completely oxidizing organic compounds to carbon dioxide coupled to $\mathrm{Fe}(\mathrm{III})$ reduction and have been found to be ubiquitous in subsurface environments [2-6]. In addition to $\mathrm{Fe}(\mathrm{III})$ reduction, Geobacter species can also reduce a variety of toxic and radioactive metals, thus can be applied to efficient bioremediation of uranium, plutonium, technetium, and vanadium [7-9]. Geobacter species can also transfer electrons to electrodes to conserve energy for growth $[10,11]$. It has been demonstrated that Geobacter sulfurreducens produces electrically conductive pili that function as nanowires to promote electron transfer to insoluble electron acceptors such as Fe(III) oxide and electrodes $[12,13]$. Therefore, Geobacter species have been utilized to harvest electricity from waste organic matter $[10,14]$ and as a biocatalyst in microbial fuel cell applications $[15,16]$.

Geobacter metallireducens was the first organism in pure culture that could oxidize organic compounds with $\mathrm{Fe}(\mathrm{III})$ oxide serving as electron acceptor $[2,17]$. This strict anaerobe can utilize a wide range of organic compounds as electron donors, including acetate, ethanol, propionate, butyrate, pyruvate, propanol, and butanol [2]. More importantly, G. metallireducens was found to completely oxidize monoaromatic compounds such as toluene, phenol, cresol, benzoate, benzaldehyde, and benzylalcohol coupled to $\mathrm{Fe}(\mathrm{III})$ reduction $[2,18]$. Several recent studies suggested a core benzoyl-CoA degradation pathway in the utilization of these aromatic compounds [19-22]. G. metallireducens can also use nitrate as electron acceptor $[2,23]$.

Constraint-based modeling enables the development of genome-scale in silico models that can predict the behavior of complex biological systems and their responses to the environments. Such a modeling approach was successfully applied to provide physiological and ecological insights on the metabolism of G. sulfurreducens [24], and has been used to optimize its applications in energy production and bioremediation [25]. Due to its wide range of electron donors and acceptors, G. metallireducens has more metabolic capabilities and therefore more potential applications than G. sulfurreducens. The genome sequence of G. metallireducens was recently completed http:// www.jgi.doe.gov/. Here, we report the development of a genome-scale metabolic model of G. metallireducens and the application of the model to study its metabolism.

\section{Methods}

\section{Metabolic network reconstruction}

The G. metallireducens metabolic network was reconstructed by a modified version of previously published procedure [26]. The reconstruction was carried out in SimPheny (Genomatica, Inc., CA) from the annotated open reading frames (ORFs) encoded in the G. metallireducens genome. The sequence similarity search (BLAST) results of the G. metallireducens genome with the genomes of several high-quality genome-scale metabolic models were utilized to create a draft model that served to accelerate the reconstruction of the genome-scale metabolic model. The reactions and genes in the draft model were manually reviewed using the gene annotations and the available biochemical and physiological information. The biomass demand reaction based on biomass composition and maintenance parameters in the published G. sulfurreducens model were used in the reconstructed G. metallireducens model. The resulting network was then subjected to the gap filling process to allow biomass formation under physiological growth conditions. For gap filling, simulations were performed to determine if the network could synthesize every single component of the biomass and the missing reactions in the pathways were identified. These reactions were reviewed for gene association, or added as non-gene associated reactions to enable the formation of biomass by the reconstructed network under physiological conditions. The reconstructed model was then used to generate a set of experimentally testable hypotheses and predictions. The experimental findings were in turn used to further refine and expand the reconstructed model in an iterative process.

\section{In silico analysis of metabolism}

The metabolic capabilities of the G. metallireducens model were calculated using flux balance analysis through linear optimization [26] in SimPheny. For growth simulations, biomass synthesis was selected as the objective function to be maximized. For energy requirement simulations, the ATP maintenance requirement reaction was selected as the objective function to be maximized. The simulations resulted in flux values in unit of $\mathrm{mmol} / \mathrm{g}$ dry weight (gdw)/h. All simulations were of anaerobic growth on minimal media, where the following external metabolites were allowed to freely enter and leave the network: $\mathrm{CO}_{2}, \mathrm{H}^{+}, \mathrm{H}_{2} \mathrm{O}, \mathrm{K}^{+}, \mathrm{Mg}^{2+}, \mathrm{NH}_{4}^{+}$, $\mathrm{PO}_{4}{ }^{3-}$, and $\mathrm{SO}_{4}{ }^{2-}$. The electron donors or electron acceptors tested were allowed a maximum uptake rate into the network as specified in the results. All other external metabolites were only allowed to leave the system. Flux variability analysis was carried out in SimPheny using method described before [27].

In silico deletion analysis was carried out for growth with acetate as the electron donor and $\mathrm{Fe}(\mathrm{III})$ or fumarate as the electron acceptor with acetate as the limiting nutrient. Maximization of biomass synthesis was the objective function. Deletions resulting in reduced growth compared to wild type were categorized as intermediate phenotype. 


\section{Strains and culture conditions}

The G. metallireducens strain used in the growth experiments was constructed with a dicarboxylic acid transporter from $G$. sulfurreducens that grew with fumarate as the sole electron acceptor [28]. The strain was cultured with appropriate electron donors in the NBAF medium that contained $4.64 \mathrm{~g} / \mathrm{l}$ fumaric acid, $0.42 \mathrm{~g} / \mathrm{l} \mathrm{KH_{2 }} \mathrm{PO}_{4}$, $0.22 \mathrm{~g} / 1 \mathrm{~K}_{2} \mathrm{HPO}_{4}, 0.20 \mathrm{~g} / 1 \mathrm{NH}_{4} \mathrm{Cl}, 0.38 \mathrm{~g} / \mathrm{l} \mathrm{KCl}, 0.36 \mathrm{~g} / \mathrm{l}$ $\mathrm{NaCl}, 0.04 \mathrm{~g} / \mathrm{l} \mathrm{CaCl}-\mathrm{H}_{2} 2 \mathrm{O}, 0.12 \mathrm{~g} / \mathrm{l} \mathrm{MgSO}_{4}-7 \mathrm{H}_{2} \mathrm{O}, 1.8 \mathrm{~g} / \mathrm{l}$ $\mathrm{NaHCO}_{3}, 0.5 \mathrm{~g} / \mathrm{l} \mathrm{Na}_{2} \mathrm{CO}_{3}-\mathrm{H}_{2} \mathrm{O}$, and $1 \mu \mathrm{M} \mathrm{Na} \mathrm{SeO}_{4}$. The NBAF medium was supplemented with $15 \mathrm{ml} / \mathrm{l}$ vitamin mixtures and $10 \mathrm{ml} / \mathrm{l}$ mineral mixtures [29,30], and was adjusted to $\mathrm{pH}$ 7.0. For growth experiments, the electron donors were added separately from the prepared stocks to the NBAF medium. Final electron donor concentration in the NBAF medium was fixed to $25 \mathrm{mM}$ for both ethanol and pyruvate.

Stock solutions of ethanol and pyruvate were prepared, filtered with $0.2 \mu \mathrm{m}$ filters, bubbled with $\mathrm{N}_{2}$, and capped separately. For the growth experiments, serum bottles containing the culture medium were flushed with $\mathrm{N}_{2}: \mathrm{CO}_{2}$ $(80 / 20)$ to remove any trace of oxygen in the bottles, capped with thick butyl-rubber stoppers, and autoclaved.

For growth with benzoate, G. metallireducens cultures were grown in triplicate at $30^{\circ} \mathrm{C}$ in anaerobic continuous culture vessels as previously described [30]. Defined, bicarbonate-buffered media with $1.0 \mathrm{mM}$ benzoate as the limiting electron donor and $\mathrm{Fe}$ (III) citrate as the electron acceptor was provided at a dilution rate of $0.05 \mathrm{~h}^{-1}$. At steady state, protein concentration was $8.2( \pm 0.2) \mathrm{mg} / \mathrm{L}$ and $\mathrm{Fe}(\mathrm{II})$ concentration was $30.3( \pm 0.4) \mathrm{mM}$. Fe(II) was determined using the ferrozine assay as previously described [31].

\section{Analytical techniques}

Samples for organic acid analysis were filtered using 0.2 $\mu \mathrm{m}$ filters and stored at $-20^{\circ} \mathrm{C}$. The samples were analyzed together using an HPLC (Dionex, Sunnyvale, CA) with a mobile phase of $0.5 \mathrm{mM} \mathrm{H}_{2} \mathrm{SO}_{4}$ at a flow rate of $0.3 \mathrm{ml} /$ min. Peaks were identified and quantified by comparing to those obtained from the standards of ethanol, pyruvate, and fumarate. HPLC data were used to estimate the time profiles of the electron donor and electron acceptor concentrations in samples of G. metallireducens in NBAF media.

\section{Results and discussion}

\section{Metabolic network reconstruction}

A draft model of G. metallireducens was built by using pairwise BLASTp comparison of the G. metallireducens genome with the genomes of the several high-quality base models in Genomatica model database including previously published G. sulfurreducens [24], Escherichia coli [26,32,33] and
Bacillus subtilis [34] models. The G. metallireducens draft model comprised 514 reactions. Among the base models used, G. sulfurreducens contributed $93 \%$ of the top BLASTp matches; this confirmed the close relationship between these two organisms. The G. metallireducens draft model captured significant portions of central metabolism, and the biosynthetic pathways for amino acids, nucleotides, and lipids.

The reactions and their gene associations in the draft model of G. metallireducens were evaluated manually based on gene annotations, published biochemical and physiological information, and external references as previously described [35]. The remaining genes were also reviewed for inclusion in the reconstructed network. A biomass demand reaction based on the combination of biomass components that were experimentally determined in G. metallireducens and represented in the published G. sulfurreducens model [24] was used in G. metallireducens model. Similarly, the energy parameters such as growth-associated energy requirements in the published G. sulfurreducens model [24] were used in the G. metallireducens model for the close relationship between these two organisms.

The unique metabolic capabilities of $G$. metallireducens to degrade monoaromatic compounds were reconstructed in the metabolic model. Monoaromatic compounds such as toluene, phenol, cresol, benzoate, benzaldehyde, and benzylalcohol are converted into benzoyl-CoA and then through the benzoyl-CoA degradation pathway to acetylCoA [19-22]. Specifically, benzylalcohol and benzaldehyde are oxidized by dehydrogenases to benzoate, which is then converted into benzoyl-CoA by benzoate CoA ligase, whereas cresol and phenol are converted to 4hydroxybenzoate and then reduced to benzoyl-CoA through 4-hydroxybenzoyl-CoA. Toluene is converted to benzoyl-CoA via benzylsuccinyl-CoA.

For gap filling, the ability of the metabolic network to synthesize a full complement of amino acids, nucleotides, lipids, carbohydrates, and cofactors from a minimal medium containing the known electron donors and acceptors was assessed. The missing reactions in the pathways were identified and reviewed. Some missing reactions were associated with G. metallireducens genes based on biochemical or genomic evidences and were included in the reconstructed network. Other missing reactions were added to the model as non-gene associated reactions to enable the reconstructed network to synthesize metabolites for biomass formation. The reconstructed network contains 30 non-gene associated reactions with different justification. These non-gene associated reactions fell into several categories: 2 reactions, 2-Oxo-4-methyl-3-carboxypentanoate decarboxylation and L-glutamate 5-semial- 
dehyde dehydratase, are non-enzymatic conversions that happen spontaneously under physiological conditions; 4 gas diffusion processes allow the transport of these gases; 1 reaction is for ATP maintenance requirement; 5 transporter reactions for electron donors ensure consistency with growth results; and 18 non-gene associated reactions are required for biomass formation under known growth conditions (see Additional file 1 for details). Non-gene associated reactions in the latter two categories are presumptive metabolic functions encoded potentially by unknown genes, and thus will be subjected for further genomic and biochemical investigation in the future.

Simulations were also utilized to understand individual reactions in the network. For example, the initial step of benzoyl-CoA degradation pathway is catalyzed by a benzoyl-CoA reductase. In Thauera aromatica, benzoyl-CoA reductase reduces the aromatic ring in two single-electron transfer steps to yield cyclohexa-1,5-diene-1-carbonylCoA with stoichiometric 2-ATP hydrolysis [36]. To understand the ATP hydrolysis stoichiometry associated with benzoyl-CoA reduction in G. metallireducens, biomass was collected from G. metallireducens cells grown with benzoate in chemostat and the experimental results were compared to simulation results where different ATP hydrolysis stoichiometry was assumed for the benzoylCoA reduction (Figure 1). The experimental growth data, a protein yield of $8.2( \pm 0.2) \mathrm{mg} / \mathrm{L}$ from $1.0 \mathrm{mM}$ benzoate at a dilution rate of $0.05 \mathrm{~h}-1$, predicted a biomass yield of $0.59( \pm 0.02) \mathrm{gdw}$ per mol of electrons at benzoate flux of $2.81 \mathrm{mmol} / \mathrm{gdw} / \mathrm{h}$ assuming $46 \%$ biomass content as pro-

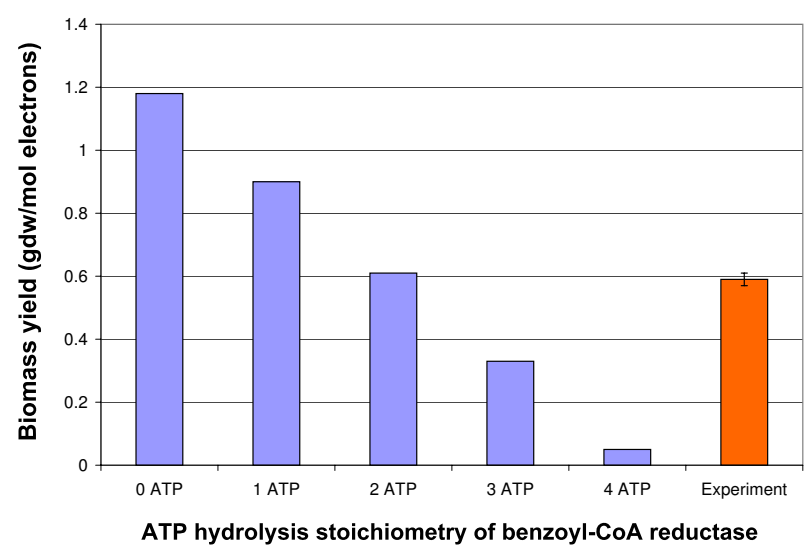

Figure I

ATP hydrolysis stoichiometry of benzoyl-CoA reduction in G. metallireducens metabolic model. BenzoylCoA reductase reactions with 0-4 ATP hydrolysis stoichiometry were applied in simulations. The predicted biomass yields as gdw/mol electrons were calculated and compared to the experimental result obtained from protein content measurement at steady state. tein. The biomass yields from a benzoate flux of 2.81 $\mathrm{mmol} / \mathrm{gdw} / \mathrm{h}$ with an ATP hydrolysis stoichiometry for benzoyl-CoA reduction between 0-4 were simulated and the in silico results were compared to the experimental result (Figure 1). As shown in Figure 1, the 2-ATP hydrolysis stoichiometry for benzoyl-CoA reduction closely matched the experimental result. Thus, the benzoyl-CoA reduction in G. metallireducens model shared the same ATP hydrolysis stoichiometry as in T. aromatica.

\section{Metabolic network of G. metallireducens}

At its completion, the manually curated genome-scale network of G. metallireducens included 747 genes of the 3389 genes in the G. metallireducens genome (Table 1). The G. metallireducens metabolic model contains 697 reactions and 769 metabolites including 58 extracellular metabolites. The detailed list of genes, reactions, metabolites, and gene-protein-reaction (GPR) associations in the metabolic model are available as supplementary information (see Additional file 2). The characteristics of the G. metallireducens model are similar to those of the updated G. sulfurreducens model (the published G. sulfurreducens model [24] was updated to incorporate the most recent results from both experimental and computational research, see Additional file 3 for detailed list of reactions). The 697 reactions of the G. metallireducens model were categorized into 9 functional groups and the results were summarized in Figure 2. Among different functional groups, reactions for biosynthesis of amino acids, lipids and cell wall components, cofactors, and nucleic acids are the most abundant, accounting for almost $70 \%$ of all the reactions. Currently, there are 76 reactions associated with transporting metabolites, including redundant transporters for the some extracellular metabolites. In addition, G. metallireducens genome contains many genes encoding components of these $\mathrm{ABC}$ transporters that are not included in the network because the substrate specificity of these $\mathrm{ABC}$

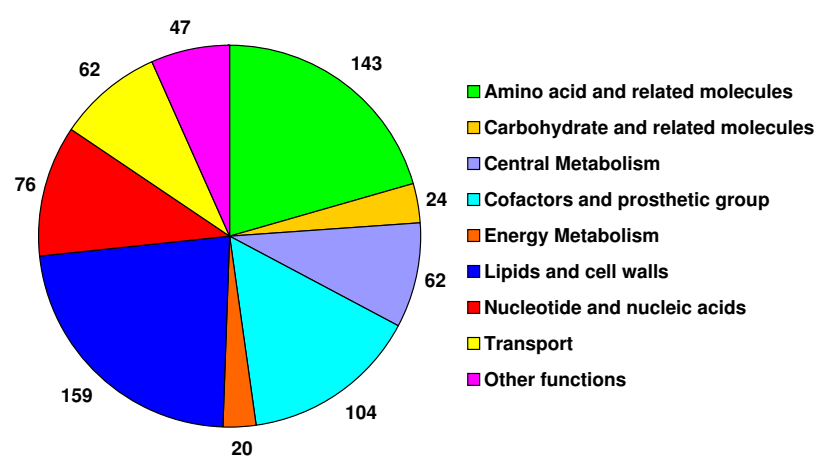

Figure 2

Functional classification of metabolic reactions in $\mathbf{G}$. metallireducens model. The 697 reactions in $G$. metallireducens model were categorized into 9 functional groups. 
Table I: Characteristics of the G. metallireducens genome-scale metabolic model compared with the G. sulfurreducens model.

\begin{tabular}{lrr}
\hline & G. metallireducens & G. sulfurreducens \\
\hline Total Genes & 3532 & 3468 \\
$\quad$ Included Genes & $747(21.2 \%)$ & $730(21.1 \%)$ \\
$\quad$ Excluded Genes & $2785(78.8 \%)$ & $2738(78.9 \%)$ \\
Total Proteins & 623 & 582 \\
Total Reactions & 697 & 649 \\
$\quad$ Non-gene Reactions & $30(3.7 \%)$ & $32(4.9 \%)$ \\
$\quad$ Input/Output Reactions & 60 & 55 \\
Total Metabolites & 769 & 698 \\
$\quad$ Extracellular Metabolites & $58(7.7 \%)$ & $55(7.9 \%)$
\end{tabular}

transporters is largely unknown. Future experiments on physiology in different environments will provide additional evidence to include these transporting systems.

To study the conservation between the G. metallireducens and G. sulfurreducens models, reactions were categorized and compared (Table 2). Overall, the two models share 579 common reactions, representing $83 \%$ of all G. metallireducens reactions and $89 \%$ of all G. sulfurreducens reactions. Among these common reactions, 140 reactions related to amino acid biosynthesis, 119 reactions in lipids and cell walls metabolism, 104 reactions of cofactor biosynthesis, and 74 reactions for nucleotide metabolism are shared between the two models, which together account for $75 \%$ of all the common reactions.

G. metallireducens can utilize a much wider range of electron donors and acceptors $[2,18,23]$ than G. sulfurreducens, which uses only acetate, $\mathrm{H}_{2}$ and lactate as the electron donors. The G. metallireducens metabolic model contains 118 unique reactions out found in the G. sulfurreducens model. Many of these unique reactions reflect of the diversity of G. metallireducens' metabolic capabilities. For example, the G. metallireducens model contains 32 unique reactions involved in the degradation pathways of aromatic compounds. G. metallireducens can also utilize several substrates other than the aromatic compounds that G. sulfurreducens does not use. G. metallireducens contains several alcohol dehydrogenase genes with substrate specificities for ethanol, propanol, and butanol that are not believed to be present in G. sulfurreducens. The enzymes coded by these genes catalyze several unique reactions that are key steps in the utilization of these alcohol substrates. The corresponding transporter reactions were also added to the G. metallireducens model, but not in the G. sulfurreducens model. Similarly, a butyrate kinase reaction unique to the G. metallireducens model allows the utilization of butyrate. These unique reactions in the $G$. metallireducens model enable the growth of the G. metallireducens model on a wide range of substrates and has accurately captured the known physiological characteristics of G. metallireducens $[2,18,23]$.

\section{In silico characterization of $\mathbf{G}$. metallireducens metabolism}

Simulations of metabolism with the G. metallireducens model were utilized to make testable predictions of $G$. metallireducens metabolism. In silico characterization of $G$. metallireducens growth with different substrates was carried out and the results are summarized in Figure 3. The

Table 2: Comparison of reactions in G. metallireducens and G. sulfurreducens metabolic models.

\begin{tabular}{|c|c|c|c|c|c|c|c|}
\hline & \multicolumn{3}{|c|}{ G. sulfurreducens } & \multicolumn{3}{|c|}{ G. metallireducens } & \multirow[b]{2}{*}{ Common } \\
\hline & All & Unique & Percentage & All & Unique & Percentage & \\
\hline Amino acid and related molecules & 148 & 8 & $5.41 \%$ & 143 & 3 & $2.10 \%$ & 140 \\
\hline Carbohydrate and related molecules & 21 & 4 & $19.05 \%$ & 24 & 7 & $29.17 \%$ & 17 \\
\hline Central Metabolism & 56 & 8 & $14.29 \%$ & 62 & 14 & $22.58 \%$ & 48 \\
\hline Cofactors and prosthetic group & 106 & 2 & $1.89 \%$ & 104 & 0 & $0.00 \%$ & 104 \\
\hline Energy Metabolism & 22 & 5 & $22.73 \%$ & 20 & 3 & $15.00 \%$ & 17 \\
\hline Lipids and cell walls & 130 & 11 & $8.46 \%$ & 159 & 40 & $25.16 \%$ & 119 \\
\hline Nucleotide and nucleic acids & 77 & 3 & $3.90 \%$ & 76 & 2 & $2.63 \%$ & 74 \\
\hline Transport & 61 & 24 & $39.34 \%$ & 62 & 25 & $40.32 \%$ & 37 \\
\hline Other functions & 28 & 5 & $17.86 \%$ & 47 & 24 & $51.06 \%$ & 23 \\
\hline Total reactions & 649 & 70 & $10.79 \%$ & 697 & 118 & $16.93 \%$ & 579 \\
\hline
\end{tabular}


growth of G. metallireducens was simulated using 9 substrates as electron donors with either $\mathrm{Fe}$ (III) or fumarate as electron acceptor and setting the electron donor or electron acceptor as the limiting factor. Under all 4 conditions, 4-cresol provided the largest biomass yield per substrate (calculated as gdw/mol substrate) for G. metallireducens growth while acetate produced the lowest biomass yield among the 9 substrates tested. Aromatic compounds generated higher biomass yield per mole of substrate than acetate and ethanol. The biomass yields for the 9 substrates under electron acceptor limiting conditions were similar to those under electron donor limiting conditions (Figure 3A\&3B, 3C\&3D), suggesting G. metallireducens might not fully utilize the excess electron donors under acceptor limiting conditions. During Fe(III) reduction, G. metallireducens had similar predicted biomass yields on pyruvate and benzoate. This is because of the energy gain associated with the conversion of pyruvate to acetyl-CoA, whereas benzoate degrades to acetyl-CoA and energy is consumed to convert acetyl-CoA to pyruvate for biomass.

When biomass yields were calculated based on acceptor consumed, pyruvate resulted in the highest biomass yield per mol of electron acceptor under all conditions, suggesting that pyruvate may have advantages over other substrates in electron acceptor limiting environments. Acetate and ethanol had similar biomass yield per electron acceptor compared to the aromatic compounds, suggesting that they may produce the same amount of biomass when limited to same amount of electron acceptors in growth medium. Therefore, the modeling study rapidly predicted the growth yields of $G$. metallireducens under varying nutrient conditions.

\section{Comparison of G. metallireducens metabolic model to G. sulfurreducens model}

G. metallireducens also contains genes for several pathways in central metabolism that do not have corresponding homologues in G. sulfurreducens. Therefore, the unique reactions associated with these genes may provide specific metabolic capacities in the G. metallireducens model. For example, G. metallireducens is known to use nitrate as an electron acceptor $[2,23]$ and the model predicts such capability. The G. metallireducens network has transporters for nitrate uptake via nitrite antiport and the nitrate reductase (cytochrome $c$ ) to reduce nitrate, which are not present in G. sulfurreducens. These two reactions together allow electrons from cytochrome $c$ to be transferred to nitrate. Nitrate is reduced and the resulting intracellular nitrite is exchanged with extracellular nitrate using the antiporter. The G. metallireducens model also contains a nitrite proton antiporter and nitrite reductase that further reduce nitrite to ammonium and allows the utilization of nitrite.
Other reactions that are not present in the G. sulfurreducens model include the glucose 6-phosphate dehydrogenase, 6-phosphogluconolactonase, and phosphogluconate dehydrogenase, which is a part of the oxidative branch of the pentose phosphate pathway. This branch provides an efficient way to produce D-ribose-5phosphate and is an important source of NADPH. However, simulations of G. metallireducens growth predict that G. metallireducens can produce D-ribose-5-phosphate by using glyceraldehyde 3-phosphate and D-fructose-6-phosphate to produce D-xylulose 5-phosphate through transketolase and transaldolase, and then converting Dxylulose 5-phosphate to D-ribose-5-phosphate, similar as simulation of G. sulfurreducens growth. Simulations also suggest that G. metallireducens can generate NADPH through isocitrate dehydrogenase (NADP) and other reactions with NADP as cofactor in a manner similar to the G. sulfurreducens network. There was no significant change in the expression levels of these genes during growth with acetate vs. benzoate couples with $\mathrm{Fe}$ (III) reduction. The exact role of this oxidative branch of pentose pathway in G. metallireducens requires further examination.

ATP-consuming futile cycles involve multiple reactions allowing the interconversion between metabolites with a net ATP consumption, and can decrease growth. However, it is hypothesized that these futile cycles balance the metabolite pools to make other key reactions thermodynamically feasible [37]. Recent ${ }^{13} \mathrm{C}$-labeling studies in $G$. metallireducens confirmed the existence of an ATP-consuming futile cycle between pyruvate and phosphoenolpyruvate [37].

The central metabolism of G. metallireducens has several reactions that are missing in G. sulfurreducens. These reactions include the acetyl-CoA synthetase (ACS), acetyl-CoA hydrolase (ACOAH), and phosphoenolpyruvate carboxylase (PPC) reactions. These reactions may be energetically inefficient because they can participate in futile cycles that drain ATP (Figure 4A). The acetate activation reaction ACS in G. metallireducens is energetically inefficient (consuming two ATP equivalents to form one acetyl-CoA), compared to the acetyl-CoA transferase (ATO) and the combined acetate kinase/phosphotransacetylase (ACK/ PTA) pathway (consuming one ATP equivalent to form one acetyl-CoA) that are present in both models. The ACOAH reaction produces zero ATP to convert acetyl-CoA to acetate and can form futile cycles with the three routes of acetate activation, namely, the ATO, the ACS and the ACK/PTA pathways. A similar futile cycle involves phosphoenolpyruvate carboxylase and phosphoenolpyruvate carboxykinase allowing ATP-consuming interconversion between phosphoenolpyruvate and oxaloacetate (Figure 4A). Model simulations also predict that increasing fluxes 

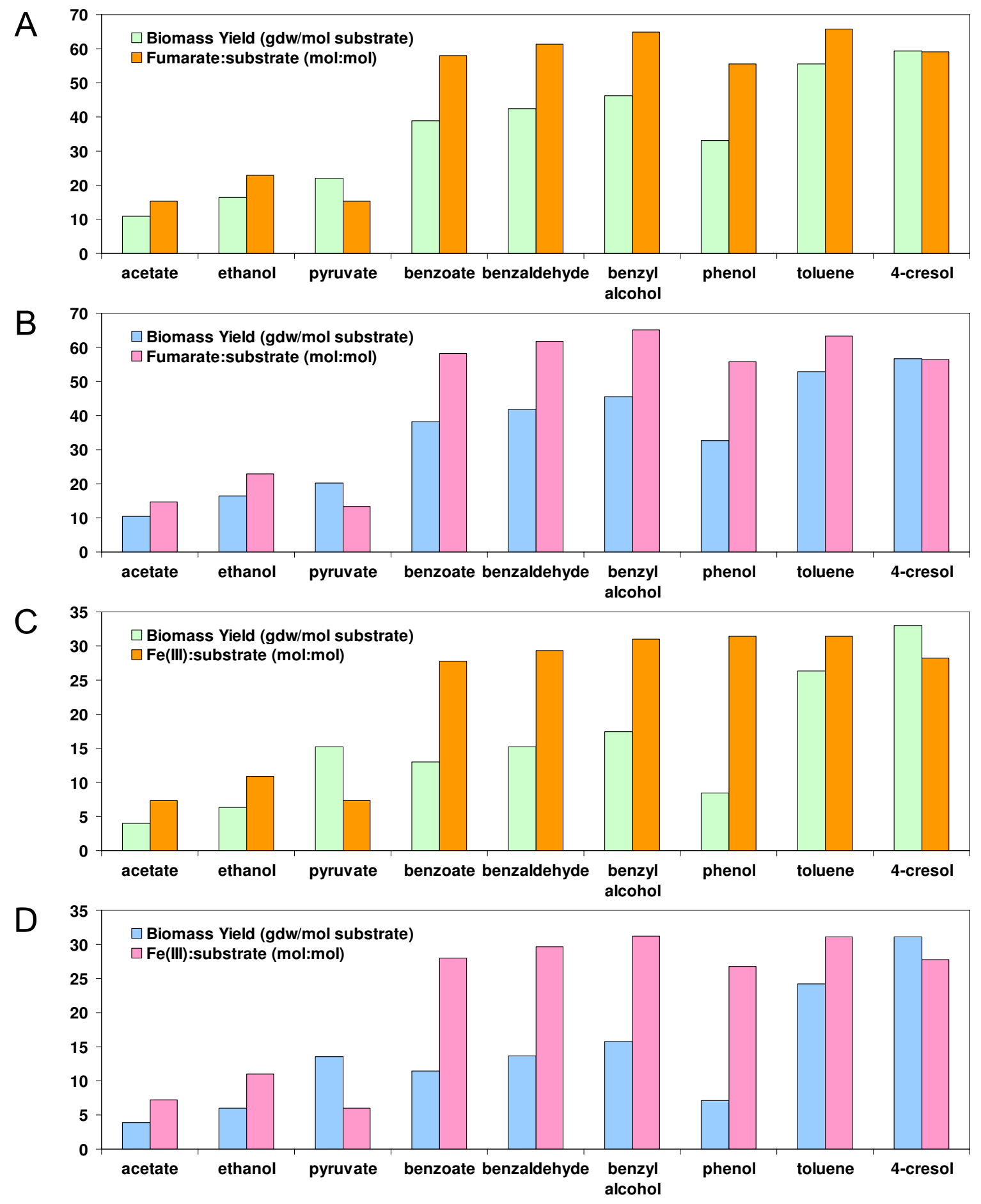

Figure 3

In silico characterization of G. metallireducens metabolism. G. metallireducens was simulated to grow with 9 different electron donors (acetate, ethanol, pyruvate, benzoate, benzaldehyde, benzyl alcohol, phenol, toluene, and 4-cresol) and 2 electron acceptors [fumarate and $\mathrm{Fe}(\mathrm{III})$ ]. The biomass yields to substrate ( $\mathrm{gdw} / \mathrm{mol}$ substrate) and the acceptor to substrate ratios $(\mathrm{mol}: \mathrm{mol})$ predicted by the model are shown in the figures. $\boldsymbol{A}$. Fumarate as the electron acceptor under donor limiting conditions; $\boldsymbol{B}$. Fumarate as the electron acceptor and the limiting nutrient; $\boldsymbol{C}$. Fe(III) as the electron acceptor under donor limiting conditions; and $\boldsymbol{D}$. Fe(III) as the electron acceptor and the limiting nutrient. 


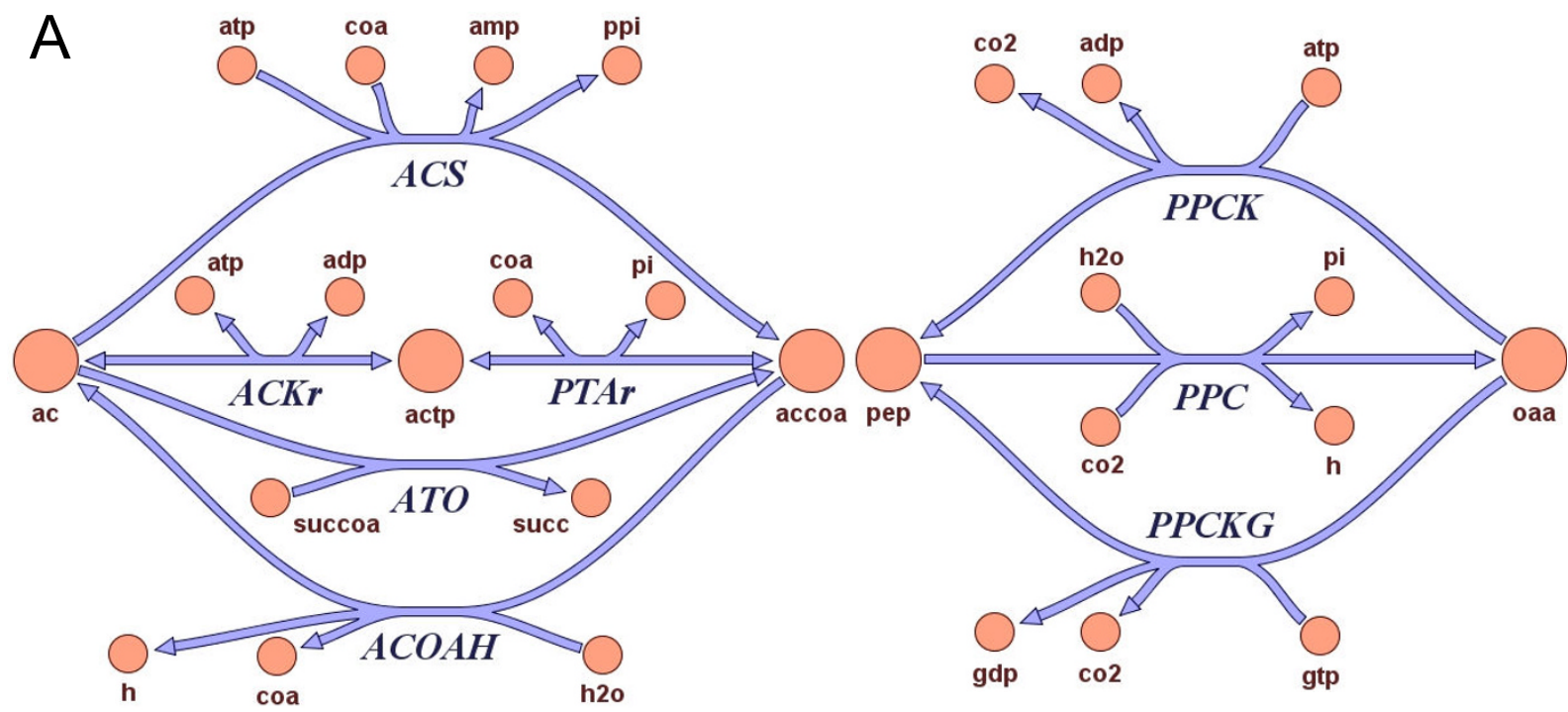

B

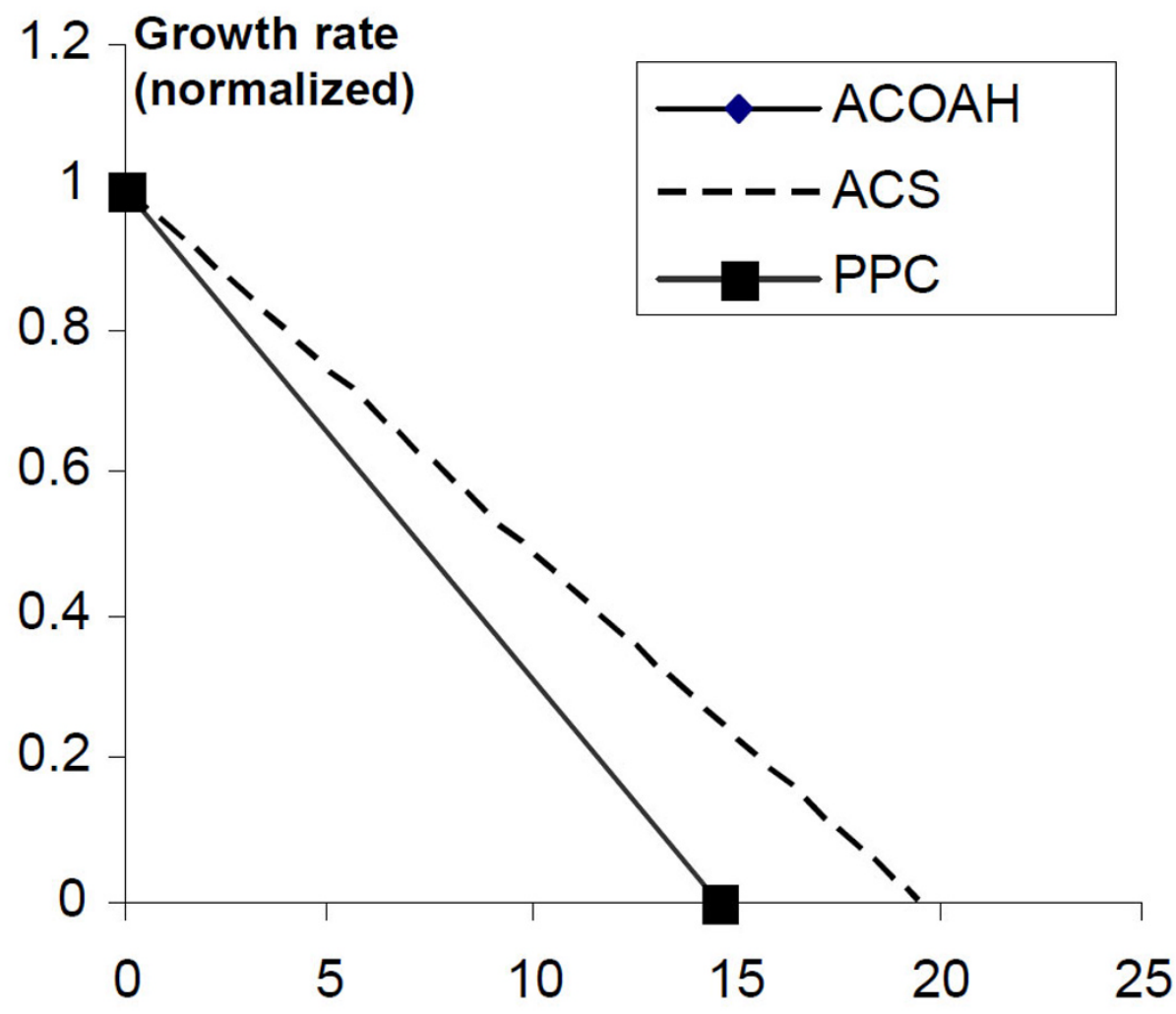

Flux Value (mmol/gdw hr.)

Figure 4

Potential futile cycles in the central metabolism of $\mathbf{G}$. metallireducens model. $A$, Two examples of energetically inefficient reactions that can form futile cycles in the central metabolism of $\boldsymbol{G}$. metallireducens model. $\boldsymbol{B}$, Effect of increasing flux through the energetically inefficient reactions on the growth rate. 
through these reactions result in an energetic penalty and consequently lowered biomass yield (Figure 4B).

\section{G. metallireducens growth with electron donors}

In order to further investigate the potential for these energetically inefficient reactions to decrease biomass yields, we measured the experimental growth of G. metallireducens in the presence of different electron donors. Unlike $G$. sulfurreducens, G. metallireducens can oxidize ethanol and pyruvate, thus enabling the further investigation of the central metabolism of the Geobacter species. In order to isolate the effect of the different electron donor oxidation pathways on the yield, a G. metallireducens strain with a dicarboxylic acid transporter that allows growth using fumarate as electron acceptor, was cultured with ethanol and pyruvate as electron donors. The G. metallireducens model simulations suggested that the biomass yield to substrate consumed (calculated as gdw/mol substrate) of pyruvate should be $34 \%$ higher than that of ethanol (Figure 5). However, the experimental results showed that similar biomass yields were obtained in pyruvate or ethanol cultures (Figure 5). HPLC measurements confirmed the complete utilization of pyruvate (data not shown). These results suggested potential energetic inefficiencies during growth with pyruvate. Most likely, the energy-inefficient reactions discussed above were active and resulted in the decreased biomass yield during growth on pyruvate.

Microarray data for the above growth conditions were not readily available. Instead, we analyzed the microarray data for G. metallireducens growing with benzoate versus

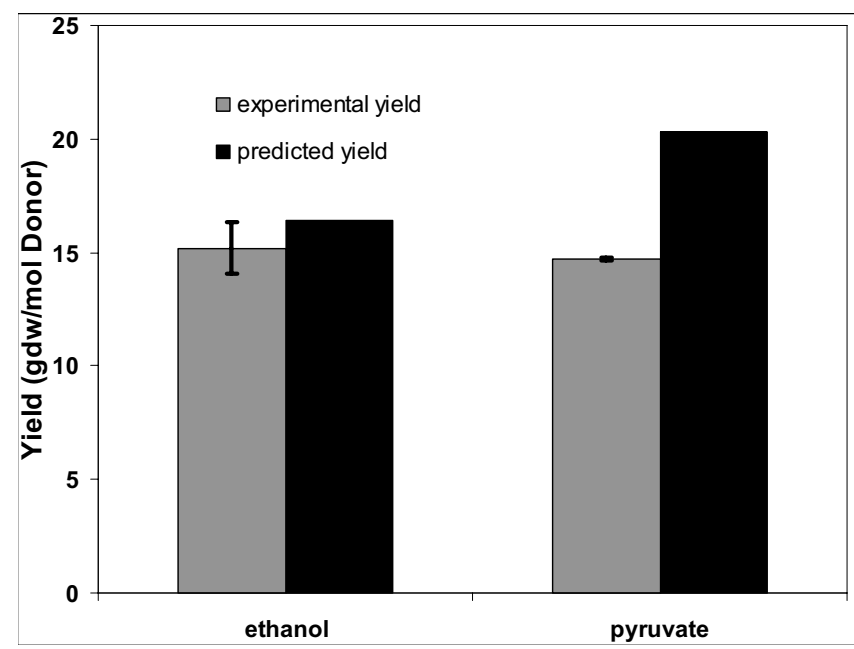

Figure 5

Comparison of experimental and predicted biomass yields. The experimental and predicted biomass yields were obtained for growth with fumarate and two different electron donors, namely, pyruvate and ethanol. acetate [21]. Among G. metallireducens genes that were significantly up-regulated ( $>50 \%$ ) by growth with benzoate versus acetate, genes encoding for ACS, PPC, and ACOAH were up-regulated by $161 \%$ to $270 \%$. The up-regulation of these genes encoding for the energy-inefficient reactions during growth with the complex substrate benzoate indicated the involvement of these energy-inefficient reactions in the metabolism of G. metallireducens when highenergy substrate benzoate is consumed. It is likely that similar up-regulation of these genes occurs during growth with pyruvate and not with ethanol.

Simulations of the G. metallireducens growth were performed using maximal biomass yield as the objective function, usually to be true in natural growth conditions where nutrients are limited. However, optimizing biomass yield may not always be the growth strategy of choice and recent studies have illustrated that under conditions of nutrient excess, maximizing the ATP production might be the chosen growth strategy [38]. Growth with high-energy substrates may also lead to a growth strategy of maximal ATP production. Under this growth strategy, the energetically inefficient reactions can be advantageous for utilizing the ATP produced. The abundance of these energetically inefficient reactions in G. metallireducens suggests that the evolution of G. sulfurreducens and G. metallireducens might have occurred in environments with different nutrient levels. In this scenario, G. sulfurreducens probably evolved in predominantly acetate limiting environments, whereas G. metallireducens probably evolved in environments with nutrient excess or with complex nutrients available.

\section{Growth simulations of $\mathbf{G}$. metallireducens using nitrate as electron acceptor}

G. metallireducens can use nitrate as an electron acceptor $[2,23]$. To better understand this capacity of nitrate respiration, G. metallireducens growth simulations were performed using nitrate as electron acceptor and acetate, ethanol, pyruvate or benzoate as electron donor. As shown in Figure 6, when nitrate was used as acceptor for G. metallireducens growth, benzoate was predicted to give the highest biomass yield to substrate (gdw/mol substrate). Pyruvate and ethanol were predicted to equally produce more biomass per substrate consumed than acetate. However, using pyruvate as substrate allowed the lowest acceptor:donor ratio, whereas benzoate had the highest. Pyruvate as substrate was predicted to have the highest biomass yield per nitrate (gdw/mol nitrate) similar to the cases discussed earlier when Fe(III) or fumarate is the electron acceptor.

Growth simulations of G. metallireducens using nitrate, fumarate or $\mathrm{Fe}$ (III) as electron acceptor were compared (Figure 7). Among the three electron acceptors, nitrate 


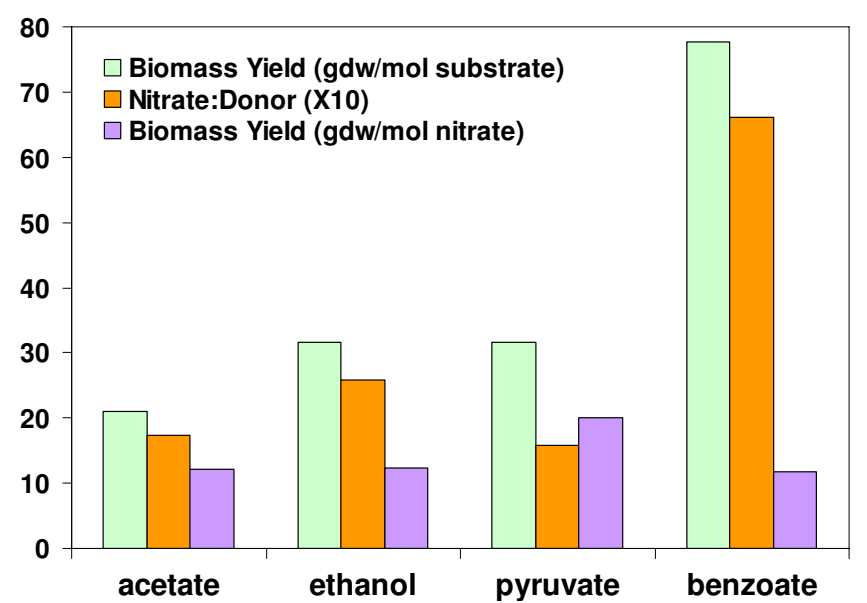

Figure 6

Simulated growth of $\mathbf{G}$. metallireducens with nitrate as electron acceptor. Growth of $G$. metallireducens was simulated using different electron donors under donor-limiting conditions. Biomass yields were predicted for both electron donor ( $\mathrm{gdw} / \mathrm{mol}$ substrate) and electron acceptor ( $\mathrm{gdw} /$ mol nitrate). The ratios of nitrate to electron donors are also shown in the figure.

resulted in the highest biomass yield per substrate consumed (gdw/mol substrate) or per electron acceptor consumed (gdw/mol electron acceptor). This is consistent with the higher energy yield coupled to nitrate reduction. The large increase in biomass yield during nitrate reduction relative to $\mathrm{Fe}(\mathrm{III})$ reduction predicts that $\mathrm{G}$. metallireducens is not limited by energy generation during nitrate reduction. For example, the fraction of the benzoate used to generate energy during nitrate reduction was predicted to be $63 \%$, compared to $94 \%$ of the benzoate used for generating energy during $\mathrm{Fe}(\mathrm{III})$ reduction. This requirement of a relatively high fraction of donor for energy generation results in higher substrate utilization rates for the same growth rate during $\mathrm{Fe}(\mathrm{III})$ reduction clearly highlighting a significant challenge associated with metal reduction.

\section{Flux distribution comparison between model prediction and ${ }^{13} \mathrm{C}$ labeling results}

${ }^{13} \mathrm{C}$ isotopomer labeling flux analysis was applied to study a simplified central metabolic network of G. metallireducens by Tang, et al. [37]. The results from that study provided an experimentally determined flux distribution. To validate the G. metallireducens model reconstructed in this work, the flux distribution results from ${ }^{13} \mathrm{C}$ isotopomer labeling flux analysis study was compared to an in silico flux distribution from the G. metallireducens model under the same acetate/ $\mathrm{Fe}(\mathrm{III})$ growth conditions $(21 \mathrm{mmol} /$ $\mathrm{gdw} / \mathrm{h}$ of acetate uptake flux with acetate as the limiting factor). Fluxes were normalized according to acetate uptake rate that was set at $100 \%$. The ${ }^{13} \mathrm{C}$ isotopomer labeling flux analysis suggested that about $90 \%$ of flux from acetyl-CoA joined the TCA cycle to produce energy and $10 \%$ of flux was routed to pyruvate and other intermediates for biomass [37]. As shown in Figure 8, model simulations predicted $91.6 \%$ of acetate was completely oxidized to $\mathrm{CO}_{2}$ via the complete TCA cycle, compared to the $90.5 \%$ from the $\left[1-{ }^{13} \mathrm{C}\right]$ acetate isotopomer labeling flux analysis. Overall, the flux distributions were similar (mean of the flux difference $=1 \% \pm 0.8 \%, \mathrm{R}^{2}=0.99$ ). The computationally predicted and experimentally determined values were well matched at high fluxes, but less consistent at low fluxes. One difference between the two analysis is that the $\left[1-{ }^{13} \mathrm{C}\right]$ acetate isotopomer labeling flux analysis used a network where serine, glycine, and cysteine were derived from 3-phosphoglycerate. However, gene encoding for these functions has not been found in G. metallireducens, whereas genes for a pathway where serine, glycine, and cysteine were derived from oxaloacetate in the TCA cycle were identified and used in G. metallireducens genome-scale model and simulations. This may account for some of the differences, such as the differences of fluxes from 3-phosphoglycerate or oxaloacetate to biomass, observed between the model simulation and the ${ }^{13} \mathrm{C}$ labeling flux analysis results.

Flux variability analysis defines a feasible range of fluxes for each individual reaction [27]. A flux variability analysis under the same constraints indicated that most flux values determined by ${ }^{13} \mathrm{C}$ labeling experiments were within such feasible ranges (data not shown), and validated the consistency between the experimental and predicted results. These results suggested that in silico growth simulation optimized for biomass formation and flux variability analysis to define the feasible flux ranges together provided a fast and easy alternative method to estimate flux distribution for the metabolism of G. metallireducens.

\section{Functional analysis of $\mathbf{G}$. metallireducens mutant phenotype}

Genome-scale metabolic model enabled the systems level gene deletion analysis for growth in defined medium. This information will provide important insight into the potential phenotypes associated with gene deletions in genetic investigations. In silico deletion analyses for $G$. metallireducens growth using electron donor/acceptor pairs of acetate/ $\mathrm{Fe}(\mathrm{III})$ or acetate/fumarate were completed and the results were shown in Figure 9. Three possible phenotypes were predicted from the deletion analysis: 1) lethal deletion with no growth observed, 2) silent mutation growing same as wild type, and 3) intermediate phenotype with reduced growth. Simulation results were the same when either $\mathrm{Fe}$ (III) or fumarate was used as electron acceptor. More than $68 \%$ of all reactions or $80 \%$ of all included genes were predicted to have no 
A
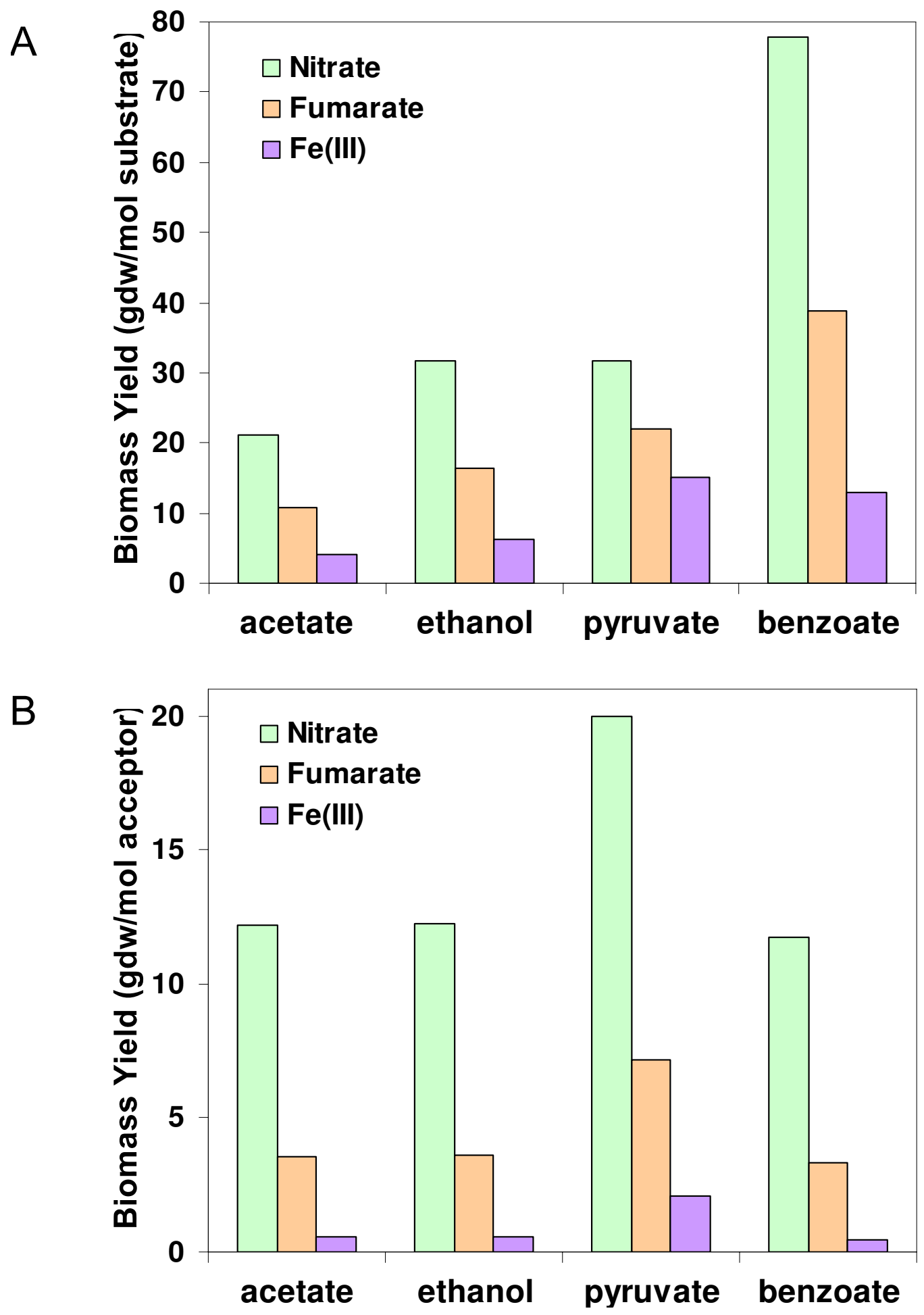

Figure 7

Comparison of G. metallireducens growth with different electron acceptors. Growth of $G$. metallireducens was simulated with the oxidation of four different electron donors (acetate, ethanol, pyruvate, and benzoate) coupled to the reduction of electron acceptors nitrate, fumarate, and $\mathrm{Fe}(\mathrm{III})$ under donor-limiting conditions. A. Predicted biomass yields to the electron donors (gdw/mol substrate) with nitrate, fumarate and $\mathrm{Fe}(\mathrm{III})$ as the acceptor; $B$. Predicted biomass yields to the electron acceptors (gdw/mol acceptor) during nitrate, fumarate and $\mathrm{Fe}(\mathrm{III})$ reduction with different electron donors. 


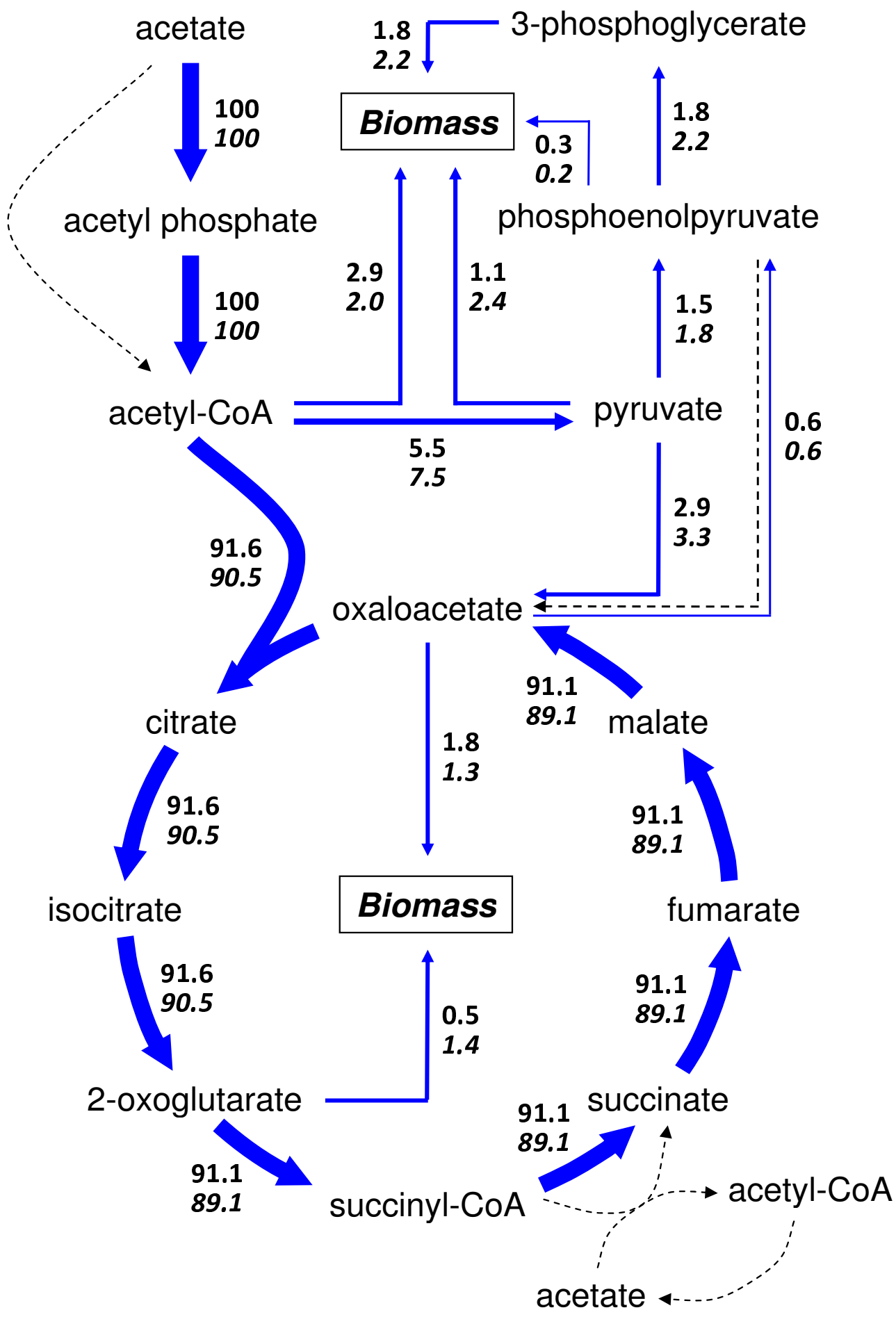

Figure 8

Metabolic flux distributions in $\mathbf{G}$. metallireducens by in silico genome-scale modeling. G. metallireducens growth with acetate as electron donor and $\mathrm{Fe}(\mathrm{III})$ as electron acceptor was simulated with under $2 \mathrm{I} \mathrm{mmol} / \mathrm{gdw} / \mathrm{h}$ of acetate uptake flux with acetate as the limiting factor, the same as a ${ }^{13} \mathrm{C}$ isotopomer labeling flux analysis. The predicted flux distributions by the genome-scale modeling (upper numbers) were compared to the flux distributions determined in the $\left[1-{ }^{13} \mathrm{C}\right]$ acetate labeling experiments (lower numbers). Dotted arrows indicated the pathways involving some energy-inefficient reactions that had no flux under this condition. 


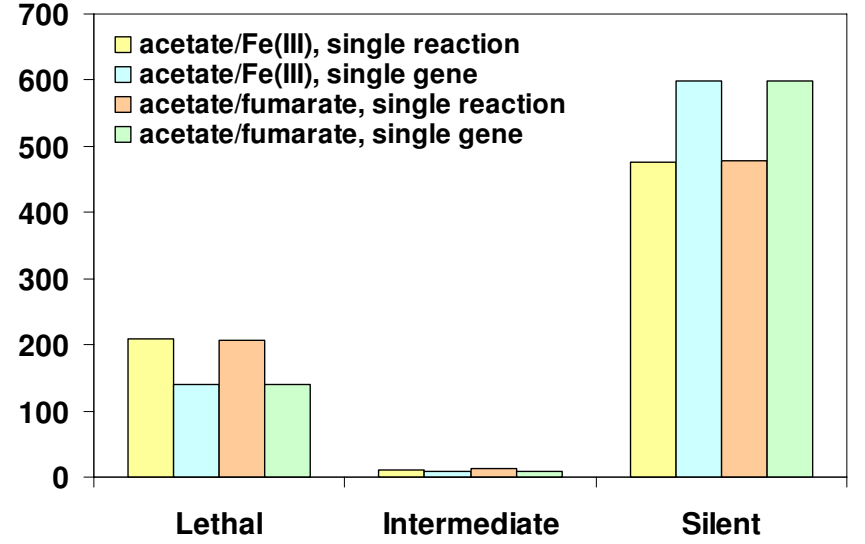

Figure 9

Functional analysis of $\mathbf{G}$. metallireducens mutant phenotype. In silico deletion analyses for $G$. metallireducens growth were completed using electron donor/acceptor pairs of acetate/Fe(III) or acetate/fumarate for every single reac-

tion or every single gene included in the genome-scale metabolic model.

effect on growth upon deletion. About $30 \%$ of all reactions and $19 \%$ of included genes were lethal mutations reflecting the inability of the perturbed network to synthesize essential components. Only for $1-2 \%$ of all reactions and included genes, deletions were predicted to have an intermediate effect on the growth rate of the G. metallireducens growth under the conditions. These deletion analysis results are similar to the results from the $G$. sulfurreducens model. This suggests that the core metabolic pathways are conserved among Geobacteraceae, and that the models of G. metallireducens and G. sulfurreducens can be used to represent of the physiology of Geobacteraceae.

\section{Conclusion}

Environmental pollution and sustainable energy are among the most important challenges that the world is facing in the 21st century. Consequently, these areas have attracted significant research efforts. In particular, Geobacter species are being extensively studied for their applications in bioremediation and bioelectricity production. However, similarities and differences in the metabolism and physiology of Geobacter species have not been well characterized. In this report, we have developed a genome-scale metabolic model for G. metallireducens to accelerate discovery and gain insight into its metabolism. Together with the published G. sulfurreducens model, the G. metallireducens metabolic model provides an important resource for the improving strategies for bioremediation and bioenergy generation.

The reconstructed metabolic model of G. metallireducens was used to gain insight into the metabolism of this bacte- rium. The G. metallireducens model is metabolically distinct from the G. sulfurreducens model, largely due to the wider range of G. metallireducens substrate utilization. The G. metallireducens metabolic model contains many additional reactions reflecting these specific metabolic capabilities that the G. sulfurreducens model does not have. In silico modeling of these additional metabolic capabilities can be used to understand how these substrates are utilized by G. metallireducens and how these capabilities can be applied in bioremediation and bioelectricity production.

Detailed examination of the G. metallireducens model suggested that its central metabolism contains several energyinefficient reactions that are not present in the G. sulfurreducens model. Experimental biomass yield of G. metallireducens growing with pyruvate was lower than the predicted optimal in silico biomass yield, and microarray data of G. metallireducens growing with benzoate and acetate indicated that genes encoding these unique reactions were up-regulated by benzoate. These results suggested that the energy-inefficient reactions were likely turned off during G. metallireducens growth with acetate to optimize biomass yield, but were up-regulated during growth with complex electron donors to improve flux for rapid energy generation. Thus, the evolution of G. sulfurreducens and G. metallireducens might have occurred in environments with different nutrient levels: $G$. sulfurreducens in a predominantly acetate limiting environments, whereas G. metallireducens probably in environments in the presence of complex nutrients or nutrient abundance. These results will help understand the physiology of these Geobacter species in the subsurface environments.

Furthermore, several in silico computational modeling approaches were applied to accelerate G. metallireducens research. For example, growth of G. metallireducens with different electron donors and electron acceptors were simulated using the genome-scale metabolic model. These simulations provided an easy and cost-effective way further understanding the metabolism of G. metallireducens. Flux distribution was compared between in silico prediction and ${ }^{13} \mathrm{C}$ labeling flux analysis results, suggesting that in silico prediction could provide a fast alternative method to estimate metabolic fluxes. Finally, the deletion analysis of the G. metallireducens metabolic model predicts phenotypes of gene knock-outs systematically and quickly. It is also important to understand that the testable hypotheses and predictions generated by in silico computational modeling with the reconstructed model should be evaluated experimentally. The experimental findings will in turn further refine and expand the reconstructed model, as well as improve our understanding of the Geobacter metabolism, in an iterative fashion.

\section{Abbreviations}

ACKr: acetate kinase; ACOAH: acetyl-CoA hydrolase; ACS: acetyl-CoA synthetase; ATO: acetyl-CoA transferase; gdw: 
gram dry weight; ORF: open reading frame; PTAr: phosphotransacetylase; PPC: phosphoenolpyruvate carboxylase; PPCK: phosphoenolpyruvate carboxykinase; PPCKG: phosphoenolpyruvate carboxykinase (GTP); ac: acetate; accoa: acetyl-CoA; actp: acetyl phosphate; oaa: oxaloacetate; pep: phosphoenolpyruvate; succ: succinate; succoa: succinyl-CoA; ORF: open reading frames; gdw: g dry weight; GPR: gene-protein-reaction.

\section{Authors' contributions}

JS, PP, TRF, and IF developed the genome-scale metabolic model of G. metallireducens. BS and JEB carried out the growth experiments. JS and RM analyzed the experimental data and drafted the manuscript. JS, CHS, DRL, and RM conceived the study and revised the manuscript. All authors read and approved the final manuscript.

\section{Additional material}

\section{Additional file 1}

Non-gene associated reactions in the G. metallireducens model. This table provides the detailed information for the non-gene associated reactions in the G. metallireducens model, including justification for the inclusion of each non-gene associated reaction in the model. Click here for file

[http://www.biomedcentral.com/content/supplementary/17520509-3-15-S1.xls]

\section{Additional file 2}

All the reactions, genes, and metabolites in the G. metallireducens model. The data provide comprehensive lists of genes, reactions, metabolites, and GPR associations in the G. metallireducens metabolic model. Click here for file

[http://www.biomedcentral.com/content/supplementary/17520509-3-15-S2.xls]

\section{Additional file 3}

All the reactions, genes, and metabolites in the G. sulfurreducens model. The data provide comprehensive lists of genes, reactions, metabolites, and GPR associations in the G. sulfurreducens metabolic model. Click here for file

[http://www.biomedcentral.com/content/supplementary/17520509-3-15-S3.xls]

\section{Acknowledgements}

This research was supported by the Office of Science (BER), Genomics:GTL Program of the U.S. Department of Energy, Grant No. DE-FC0202ER63446. We thank Olivia Bui for drawing a metabolic map for the model, and John $\mathrm{D}$. Trawick for critical reading of the manuscript.

\section{References}

I. Lovley DR, Phillips EJ: Novel Mode of Microbial Energy Metabolism: Organic Carbon Oxidation Coupled to Dissimilatory Reduction of Iron or Manganese. Appl Environ Microbiol 1988, 54: $1472-\mid 480$.

2. Lovley DR, Giovannoni SJ, White DC, Champine JE, Phillips EJ, Gorby YA, Goodwin S: Geobacter metallireducens gen. nov. sp. nov., a microorganism capable of coupling the complete oxidation of organic compounds to the reduction of iron and other metals. Arch Microbiol 1993, 159:336-344.

3. Caccavo F Jr, Lonergan DJ, Lovley DR, Davis M, Stolz JF, Mclnerney MJ: Geobacter sulfurreducens sp. nov., a hydrogen- and acetate-oxidizing dissimilatory metal-reducing microorganism. Appl Environ Microbiol 1994, 60:3752-3759.

4. Lovley DR, Holmes DE, Nevin KP: Dissimilatory Fe(III) and Mn(IV) reduction. Adv Microb Physiol 2004, 49:219-286.

5. Nevin KP, Holmes DE, Woodard TL, Hinlein ES, Ostendorf DW, Lovley DR: Geobacter bemidjiensis sp. nov. and Geobacter psychrophilus sp. nov., two novel Fe(III)-reducing subsurface isolates. Int J Syst Evol Microbiol 2005, 55:1667-1674.

6. Shelobolina ES, Nevin KP, Blakeney-Hayward JD, Johnsen CV, Plaia TW, Krader P, Woodard T, Holmes DE, Vanpraagh CG, Lovley DR: Geobacter pickeringii sp. nov., Geobacter argillaceus sp. nov. and Pelosinus fermentans gen. nov., sp. nov., isolated from subsurface kaolin lenses. Int / Syst Evol Microbiol 2007, 57: I 26- I 35.

7. Lloyd JR, Sole VA, Van Praagh CV, Lovley DR: Direct and Fe(II)mediated reduction of technetium by $\mathrm{Fe}$ (III)-reducing bacteria. Appl Environ Microbiol 2000, 66:3743-3749.

8. Lloyd JR, Chesnes J, Glasauer S, Bunker DJ, Livens FR, Lovley DR: Reduction of actinides and fission products by $\mathrm{Fe}$ (III)-reducing bacteria. Geomicrobiology Journal 2002, 19:103-120.

9. Ortiz-Bernad I, Anderson RT, Vrionis HA, Lovley DR: Vanadium respiration by Geobacter metallireducens: novel strategy for in situ removal of vanadium from groundwater. Appl Environ Microbiol 2004, 70:3091-3095.

10. Bond DR, Holmes DE, Tender LM, Lovley DR: Electrode-reducing microorganisms that harvest energy from marine sediments. Science 2002, 295:483-485.

II. Bond DR, Lovley DR: Electricity production by Geobacter sulfurreducens attached to electrodes. Appl Environ Microbiol 2003, 69:1548-1555.

12. Reguera G, McCarthy KD, Mehta T, Nicoll JS, Tuominen MT, Lovley DR: Extracellular electron transfer via microbial nanowires. Nature 2005, 435: 1098-II0I.

13. Reguera G, Nevin KP, Nicoll JS, Covalla SF, Woodard TL, Lovley DR: Biofilm and nanowire production leads to increased current in Geobacter sulfurreducens fuel cells. Appl Environ Microbiol 2006 , 72:7345-7348.

14. Holmes DE, Bond DR, O'Neil RA, Reimers CE, Tender LR, Lovley $D R$ : Microbial communities associated with electrodes harvesting electricity from a variety of aquatic sediments. Microb Ecol 2004, 48: $178-190$.

15. Lovley DR: Bug juice: harvesting electricity with microorganisms. Nat Rev Microbiol 2006, 4:497-508.

16. Lovley DR: Microbial fuel cells: novel microbial physiologies and engineering approaches. Curr Opin Biotechnol 2006, I7:327-332.

17. Lovley DR, Baedecker M, Lonergan DJ, Cozzarelli I, Phillips EJP, Siegel D: Oxidation of aromatic contaminants coupled to microbial iron reduction. Nature 1989, 339:297-300.

18. Lovley DR, Lonergan DJ: Anaerobic Oxidation of Toluene, Phenol, and p-Cresol by the Dissimilatory Iron-Reducing Organism, GS-1 5. Appl Environ Microbiol 1990, 56:1858-1864.

19. Kane SR, Beller HR, Legler TC, Anderson RT: Biochemical and genetic evidence of benzylsuccinate synthase in toluenedegrading, ferric iron-reducing Geobacter metallireducens. Biodegradation 2002, I 3: I49-I54.

20. Wischgoll S, Heintz D, Peters F, Erxleben A, Sarnighausen E, Reski R, van Dorsselaer A, Boll M: Gene clusters involved in anaerobic benzoate degradation of Geobacter metallireducens. Mol Microbiol 2005, 58: I238-1252.

2I. Butler JE, He Q, Nevin KP, He Z, Zhou J, Lovley DR: Genomic and microarray analysis of aromatics degradation in Geobacter metallireducens and comparison to a Geobacter isolate from a contaminated field site. BMC Genomics 2007, 8: 180 .

22. Peters F, Heintz D, Johannes J, van Dorsselaer A, Boll M: Genes, enzymes, and regulation of para-cresol metabolism in Geobacter metallireducens. J Bacteriol 2007, 189:4729-4738.

23. Senko JM, Stolz JF: Evidence for iron-dependent nitrate respiration in the dissimilatory iron-reducing bacterium Geobacter metallireducens. Appl Environ Microbiol 200I, 67:3750-3752.

24. Mahadevan R, Bond DR, Butler JE, Esteve-Nunez A, Coppi MV, Palsson $\mathrm{BO}$, Schilling $\mathrm{CH}$, Lovley DR: Characterization of metabolism in the $\mathrm{Fe}(\mathrm{III})$-reducing organism Geobacter 
sulfurreducens by constraint-based modeling. Appl Environ Microbiol 2006, 72:1558-1568.

25. Izallalen M, Mahadevan R, Burgard A, Postier B, Didonato R Jr, Sun J, Schilling $\mathrm{CH}$, Lovley DR: Geobacter sulfurreducens strain engineered for increased rates of respiration. Metab Eng 2008, 10:267-275.

26. Edwards JS, Palsson BO: The Escherichia coli MGI655 in silico metabolic genotype: its definition, characteristics, and capabilities. Proc Natl Acad Sci USA 2000, 97:5528-5533.

27. Reed JL, Palsson BO: Genome-scale in silico models of $E$. coli have multiple equivalent phenotypic states: assessment of correlated reaction subsets that comprise network states. Genome Res 2004, 14: 1797-1805.

28. Butler JE, Glaven RH, Esteve-Nunez A, Nunez C, Shelobolina ES, Bond DR, Lovley DR: Genetic characterization of a single bifunctional enzyme for fumarate reduction and succinate oxidation in Geobacter sulfurreducens and engineering of fumarate reduction in Geobacter metallireducens. I Bacteriol 2006, 188:450-455.

29. Esteve-Nunez A, Nunez C, Lovley DR: Preferential Reduction of Fe(III) over Fumarate by Geobacter sulfurreducens. J Bacteriol 2004, 186:2897-2899.

30. Esteve-Nunez A, Rothermich A, Sharma M, Lovley DR: Growth of Geobacter sulfurreducens Under Nutrient-Limiting Conditions in Continuous Culture. Environmental Microbiology 2005, 7:64I-648

31. Lovley DR, Phillips EJ: Rapid Assay for Microbially Reducible Ferric Iron in Aquatic Sediments. Appl Environ Microbiol 1987, 53:1536-1540.

32. Reed JL, Vo TD, Schilling CH, Palsson BO: An expanded genomescale model of Escherichia coli K-I2 (iJR904 GSM/GPR). Genome Biol 2003, 4:R54.

33. Feist AM, Henry CS, Reed JL, Krummenacker M, Joyce AR, Karp PD, Broadbelt LJ, Hatzimanikatis V, Palsson BO: A genome-scale metabolic reconstruction for Escherichia coli K-12 MG I655 that accounts for 1260 ORFs and thermodynamic information. Mol Syst Biol 2007, 3:121.

34. Oh YK, Palsson BO, Park SM, Schilling CH, Mahadevan R: Genomescale reconstruction of metabolic network in Bacillus subtilis based on high-throughput phenotyping and gene essentiality data. J Biol Chem 2007, 282(39):2879|-9.

35. Covert MW, Schilling CH, Famili I, Edwards JS, Goryanin II, Selkov E, Palsson BO: Metabolic modeling of microbial strains in silico. Trends Biochem Sci 200I, 26:179-186.

36. Boll M, Albracht SS, Fuchs G: Benzoyl-CoA reductase (dearomatizing), a key enzyme of anaerobic aromatic metabolism. A study of adenosinetriphosphatase activity, ATP stoichiometry of the reaction and EPR properties of the enzyme. Eur J Biochem 1997, 244:840-85 I.

37. Tang YJ, Chakraborty R, Martin HG, Chu J, Hazen TC, Keasling JD: Flux analysis of central metabolic pathways in Geobacter metallireducens during reduction of soluble Fe(III)-nitrilotriacetic acid. Appl Environ Microbiol 2007, 73:3859-3864.

38. Schuetz R, Kuepfer L, Sauer U: Systematic evaluation of objective functions for predicting intracellular fluxes in Escherichia coli. Mol Syst Biol 2007, 3:1 19.
Publish with Bio Med Central and every scientist can read your work free of charge

"BioMed Central will be the most significant development for disseminating the results of biomedical research in our lifetime. "

Sir Paul Nurse, Cancer Research UK

Your research papers will be:

- available free of charge to the entire biomedical community

- peer reviewed and published immediately upon acceptance

- cited in PubMed and archived on PubMed Central

- yours - you keep the copyright
BioMedcentral 